\title{
Noise Suppression and Contrast Enhancement via Bayesian Residual Transform (BRT) in Low-Light Conditions
}

Audrey G. Chung

Alexander Wong
University of Waterloo, ON, Canada

University of Waterloo, ON, Canada

\section{Abstract}

Very low-light conditions are problematic for current robotic vision algorithms as captured images are subject to high levels of ISO noise. We propose a Bayesian Residual Transform (BRT) model for joint noise suppression and image enhancement for images captured under these low-light conditions via a Bayesian-based multiscale image decomposition. The BRT models a given image as the sum of residual images, and the denoised image is reconstructed using a weighted summation of these residual images. We evaluate the efficacy of the proposed BRT model using the VIP-LowLight dataset, and preliminary results show a notable visual improvement over state-of-the-art denoising methods.

\section{Introduction}

Robotic vision is a popular field of research due to the crucial role it plays in all vision-based tasks. While there have been numerous recent advances, robotic vision systems typically encounter difficulties given practical environments, particularly in very low-light conditions. This is problematic as low-light robotic vision is essential for many applications such as night-time robotics, navigation, and computer-aided search and rescue missions.

Image noise is typically caused by the thermal noise from the camera's circuitry, and becomes particularly noticeable in low-light conditions [1, 2]. Caused by the gain (ISO) settings used in dim lighting, high levels of ISO noise are common in low-light conditions given the relatively low signal levels of the captured image. Thus, the image restoration problem is a fast growing field of research [3, 4]. Current image restoration methods include edgepreserving bilateral filtering [5], non-local means filtering [3], blockmatching 3D filtering [4], and transform-based methods such as orthonormal wavelet denoising $[6,7]$.

These state-of-the-art methods, however, tend to use patchbased approaches when facing the image restoration problem. In particular, [5] and [4] are based on local relationships, and generally have difficulty reconstructing the underlying image structures in the presence of high levels of noise. Additionally, none of these methods seek to denoise the image while simultaneously boosting the contrast. We propose a Bayesian Residual Transform (BRT) model for joint noise suppression and contrast enhancement for images contaminated with heavy noise.

\section{Methodology}

To suppress the inherent ISO noise and boost contrast, the lowlight image is first decomposed on multiple scales using the Bayesian Residual Transform (BRT) [8]. The BRT of an image, $f(\underline{x})$, is modelled as a summation of $n$ residual images:

$$
\begin{aligned}
f(\underline{x}) & =\sum_{i=1}^{n} r_{i}(\underline{x}) \\
& =f_{\sum, 1}(\underline{x})
\end{aligned}
$$

where each residual image, $r_{i}(\underline{x})$, characterizes image information at the $i^{\text {th }}$ scale. An image $f_{\Sigma, j}(\underline{x})$, then, represents the summation of residual images of scales $[j, n]$ :

$$
f_{\sum, j}(\underline{x})=\sum_{i=j}^{n} r_{i}(\underline{x})
$$

Since we can rewrite the summation as

$$
f_{\sum, 1}(\underline{x})=f_{\sum, 2}(\underline{x})+r_{1}(\underline{x}),
$$

it becomes clear that the residual image $r_{1}(\underline{x})$ represents the residual between the summation of residual images of scales $[1, n]$ and the summation of residual images of scales $[2, n]$.

Similarly to Wong et al. [8], we formulate the solution to the inverse problem of estimating $f_{\sum, 2}(\underline{x})$ given $f_{\Sigma, 1}(\underline{x})$ using the conditional expectation $E\left(f_{\Sigma, 2}(\underline{x}) \mid f_{\Sigma, 1}(\underline{x})\right)$ [9], i.e.

$$
\hat{f}_{\sum, 2}(\underline{x})=E\left(f_{\sum, 2}(\underline{x}) \mid f_{\sum, 1}(\underline{x})\right) .
$$

This can then be generalized for any $r_{j}(\underline{x})$ at scale $j$ for $j<n$ as follows:

$$
r_{j}(\underline{x})=\hat{f}_{\sum, j+1}(\underline{x})-\hat{f}_{\sum, j}(\underline{x}),
$$

where

$$
\hat{f}_{\sum, j+1}(\underline{x})=E\left(f_{\sum, j+1}(\underline{x}) \mid \hat{f}_{\sum, j}(\underline{x})\right)
$$

and $r_{n}(\underline{x})$ is simply $\hat{f}_{\sum, n}(\underline{x})$.

Since the low-light image is represented as the sum of residual images at all scales, we can assume that the residual images at certain scales are more strongly representative of the captured ISO noise. As such, we can reconstruct the low-light image with suppressed noise and boosted contrast via a weighted sum:

$$
f^{\prime}(\underline{x})=\sum_{i=1}^{n} \alpha_{i} r_{i}(\underline{x})
$$

where $\alpha_{i}$ is a weighting coefficient for the residual image at the $i^{t h}$ scale. To suppress ISO noise and enhance image contrast, the weighting coefficients (determined empirically) for residual images strongly associated with ISO noise and image content are decreased and increased, respectively.

Figure 1 shows a visualization of the joint noise suppression and contrast enhancement method via the BRT model. The image $f(x)$ is decomposed into multi-scale residual images, and recombined via a weighted summation to form the final denoised and contrast enhanced image.

\section{Results}

The proposed BRT model and state-of-the-art methods were compared using the VIP-LowLight ${ }^{1}$ dataset (as shown in Figure 2), a set of natural images captured in very low-light conditions, to investigate the real-world applicability of the methods. The dynamic range of the images were scaled to show the image content and the corresponding amplification of the ISO noise. As there was no ground truth for these images, the restored images generated by the different state-of-the-art methods and the BRT model were compared visually.

Figure 3 shows samples of captured and restored images using VIP-LowLight. The bilateral filter and the SURE-LET approach have some success suppressing the amplified ISO noise; however, the noise is still noticeable in flat areas and the images retain their grainy texture. BM3D and NLM appear to be able to eliminate more of the ISO noise in the camera images captured at low-lighting conditions, but there is noticeable distortion at the edges due to the filtering. The proposed BRT model enhances important details and textures while significantly reducing the perceived noise, and there is a notable reduction in the grainy texture of the images. 


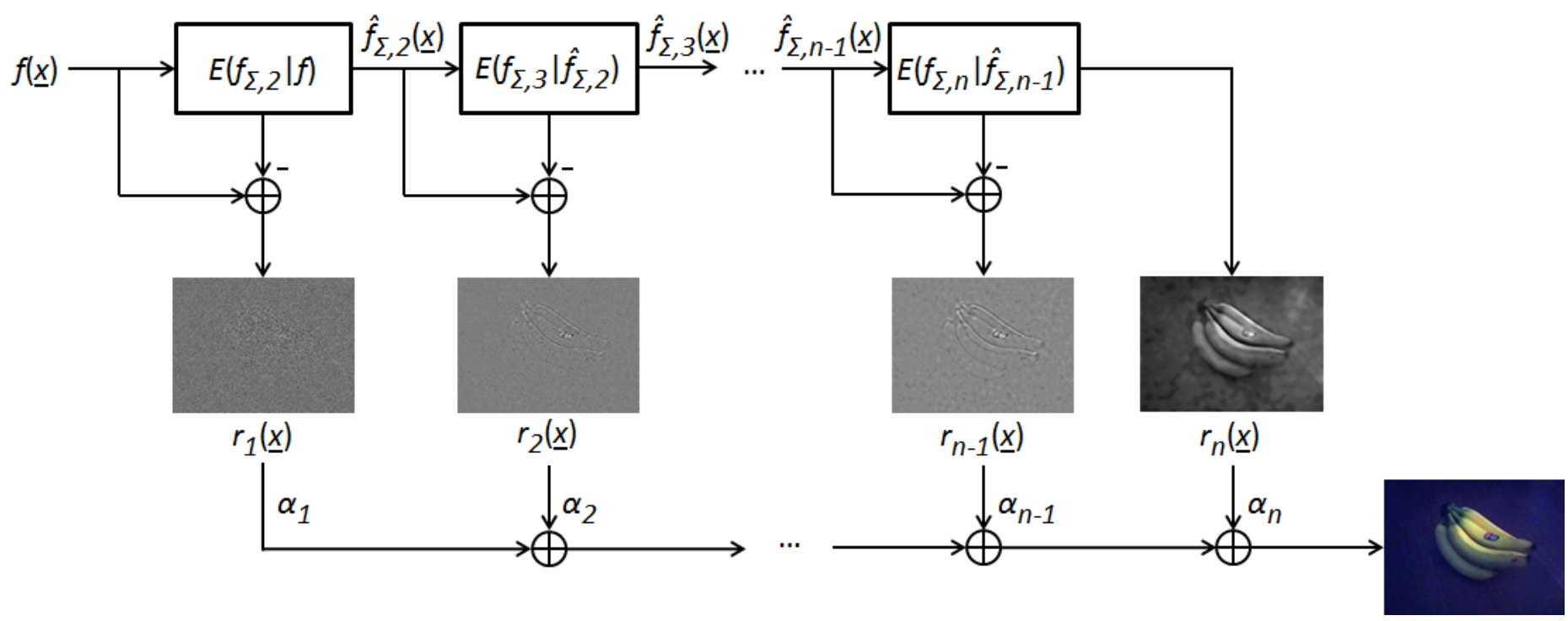

Fig. 1: Algorithm overview for noise suppression and contrast enhancement using the Bayesian Residual Transform (BRT) model. The image is decomposed into multi-scale residual images, and recombined via a weighted summation.
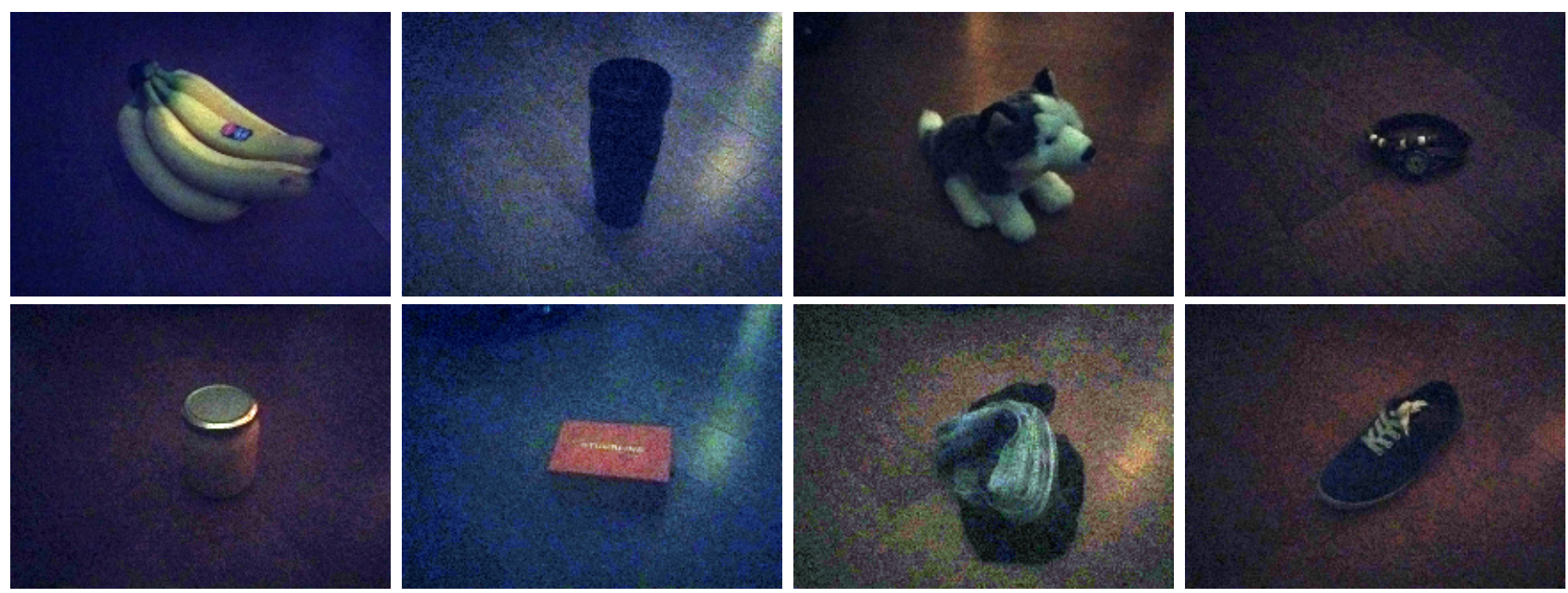

Fig. 2: Images from the VIP-LowLight database, a set of full-sized real camera data captured under very low-light conditions.

\section{Discussion}

The proposed BRT model allows for a noticeable reduction in perceived ISO noise in homogeneous areas while retaining edges associated with image texture. Though there is a significant visual improvement over the other state-of-the-art methods, the proposed BRT model still suffers from blurriness around low contrast edges, and artifact patterns similar to the ones seen in images filtered using BM3D [4]. Further investigation into the BRT model parameters (e.g., number of decomposition scales, recombination weighting coefficients) is required to fully realize an optimal noise suppression and contrast enhancement model, and testing using a larger dataset is needed to better characterize the advantages and disadvantages of the proposed BRT model.

\section{Acknowledgments}

This research has been supported by the Canada Research Chairs Program, Natural Sciences and Engineering Research Council of Canada (NSERC), and the Ministry of Research and Innovation of Ontario.

\section{References}

[1] E. Abreu, M. Lightstone, S. K. Mitra, and K. Arakawa, "A new efficient approach for the removal of impulse noise from highly corrupted images," Image Processing, IEEE Transactions on, vol. 5, no. 6, pp. 1012-1025, 1996.
[2] J. Goel, D. Chan, and P. Mandl, "Pre-processing for mpeg compression using adaptive spatial filtering," Consumer Electronics, IEEE Transactions on, vol. 41, no. 3, pp. 687-689, 1995.

[3] A. Buades, B. Coll, and J.-M. Morel, "A non-local algorithm for image denoising," in Computer Vision and Pattern Recognition, 2005. CVPR 2005. IEEE Computer Society Conference on, vol. 2. IEEE, 2005, pp. 60-65.

[4] K. Dabov, A. Foi, V. Katkovnik, and K. Egiazarian, "Image denoising by sparse 3-d transform-domain collaborative filtering," Image Processing, IEEE Transactions on, vol. 16, no. 8, pp. 2080-2095, 2007.

[5] C. Tomasi and R. Manduchi, "Bilateral filtering for gray and color images," in Computer Vision, 1998. Sixth International Conference on. IEEE, 1998, pp. 839-846.

[6] F. Luisier, T. Blu, and M. Unser, "A new sure approach to image denoising: Interscale orthonormal wavelet thresholding," Image Processing, IEEE Transactions on, vol. 16, no. 3, pp. 593-606, 2007.

[7] T. Blu and F. Luisier, "The sure-let approach to image denoising," Image Processing, IEEE Transactions on, vol. 16, no. 11, pp. 2778$2786,2007$.

[8] A. Wong and X. Y. Wang, "A Bayesian Residual Transform for Signal Processing," IEEE Access, vol. 3, pp. 709-717, 2015.

[9] P. Fieguth, Statistical Image Processing and Multidimensional Modeling. New York, NY, USA: Springer-Verlag, 2010. 


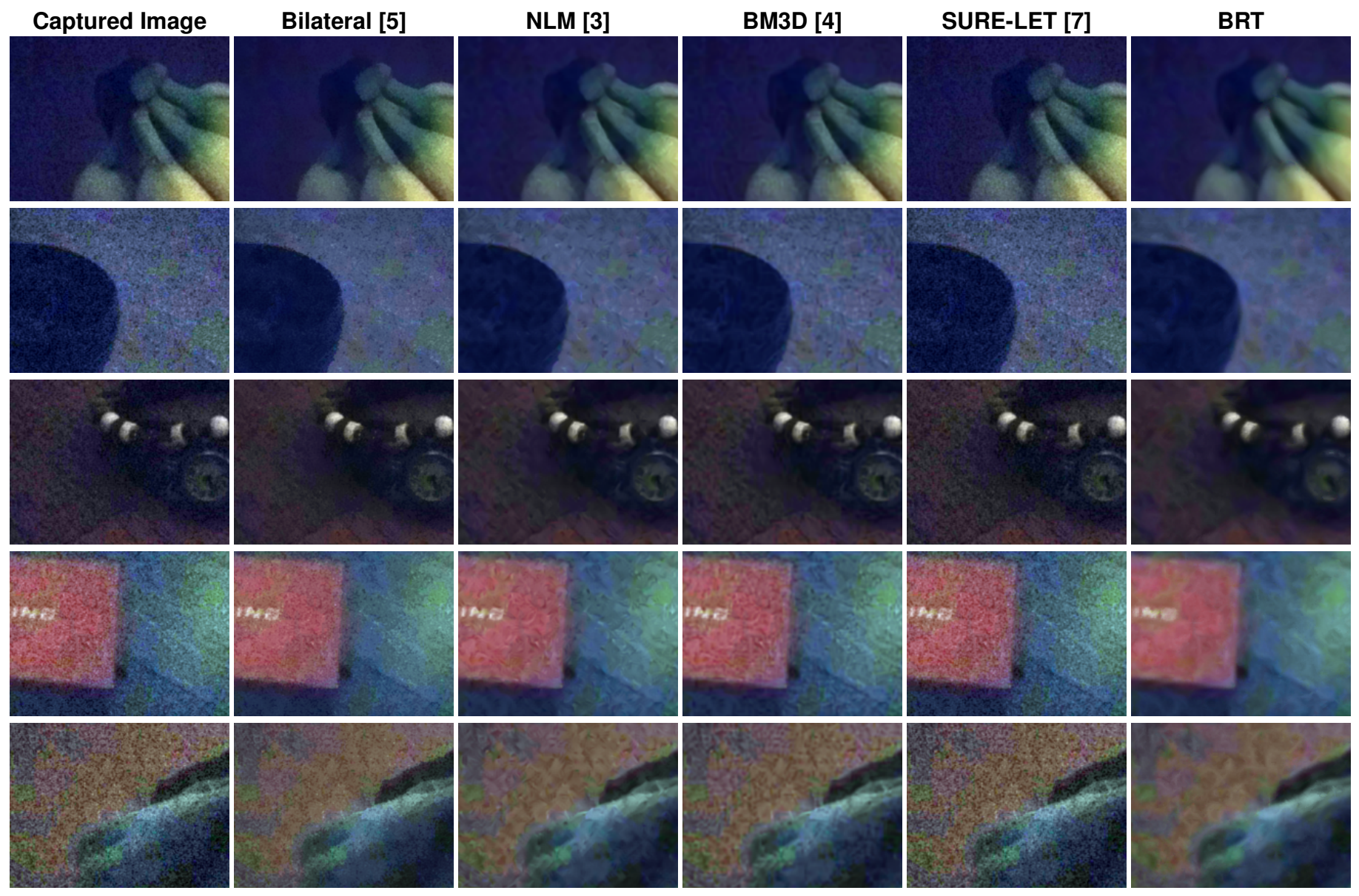

Fig. 3: Visual comparison of the bilateral filter [5], the non-local means (NLM) filter [3], the block-matching with collaborative 3-D (BM3D) filter [4], the SURE-LET approach [7], and the proposed BRT model using the VIP-LowLight dataset of real camera data captured under very low-light conditions. The images have been cropped to better illustrate the differences between the restored images (see Figure 2 for full sized captured data). 